\title{
Tratamiento laparoscópico del sindrome de la vena ovárica
}

\author{
N. del Valle González, J. Estébanez Zarranz, T. Escudero Caro*, F. Castroviejo Royo, \\ M. Mendo González*, M. Cepeda Delgado, J.Mª Martínez-Sagarra Oceja
}

Servicios de Urología y Radiodiagnóstico*. Hospital Universitario Pío del Río Hortega. Valladolid.

Actas Urol Esp 2006; 30 (1): 85-89

\section{RESUMEN \\ TRATAMIENTO LAPAROSCÓPICO DEL SÍNDROME DE LA VENA OVÁRICA}

Objetivo: Presentamos un caso de síndrome de la vena ovárica, describimos su presentación clínica y discutimos su diagnóstico y tratamiento incluyendo la cirugía laparoscópica.

Material y Métodos: Mujer de 36 años de edad con clínica de cólico renal derecho recurrente que tras ser estudiada mediante radiología de abdomen, urografías, TAC, RM y ecografía es diagnosticada finalmente de síndrome de la vena ovárica. El caso se resuelve con cirugía laparoscópica.

Conclusiones: El síndrome de la vena ovárica es una entidad poco frecuente. El diagnóstico diferencial se debe hacer con procesos extrínsecos que producen obstrucción ureteral. El tratamiento es quirúrgico cuando produce síntomas y pensamos que actualmente debe hacerse por vía laparoscópica.

Palabras clave: Vena ovárica. Obstrucción ureteral. Cirugia laparoscópica.

\section{ABSTRACT \\ LAPAROSCOPIC TREATMENT OF OVARIAN VEIN SYNDROME}

Objective: We report a case of Ovarian Vein Syndrome, describe its clinical symptoms and discuss its diagnosis and management including laparoscopic surgery treatment.

Materials and Methods: A 36-year-old female with right kidney recurring pain was studied by means of abdominal RX, urography, CT, MRI and ultrasonography and finally diagnosed from Ovarian Vein Syndrome. The case was resolved with laparoscopic surgery.

Conclusions: Ovarian Vein Syndrome is an uncommon disorder. Differential diagnosis must be done with external processes that cause ureteral obstruction. Surgery is the first line treatment when clinical symptoms are present and, in our opinion, laparoscopic surgery is the best approach to treat this pathology.

Keywords: Ovarian vein. Ureteral obstruction. Laparoscopic surgery.

$\mathrm{L}$ as venas ováricas consisten en múltiples canaMes venosos pequeños. Las ramas venosas reciben tributarias uterinas y se fusionan para formar una vena ovárica única antes de atravesar el reborde de la pelvis. En su transcurso por los vasos iliacos cruzan por delante del uréter para desembocar la del lado derecho en la vena cava a nivel de la tercera vértebra lumbar y la del lado izquierdo en la vena renal izquierda. Estas relaciones anatómicas convierten a las venas ováricas en una posible causa de obstrucción ureteral ${ }^{1}$, sien- do más frecuente la afectación del uréter derecho salvo en el caso de patologias que determinen estasis en la vena renal izquierda ${ }^{2}$.

$\mathrm{El}$ síndrome de la vena ovárica es una patología poco frecuente, fue descrito por $\mathrm{Clark}^{3}$ en 1964.

Los síntomas se suelen presentar durante el embarazo, generalmente en multíparas, aunque también pueden aparecer después del embarazo; no es infrecuente que el cuadro sintomático se agrave durante la menstruación. Los síntomas de 
presentación pueden estar relacionados con infecciones urinarias de repetición. La mayoría de las pacientes refieren dolor lumbar derecho.

Presentamos un caso de síndrome de vena ovárica en una mujer joven no gestante con clínica de cólico renal derecho de varios meses de evolución que se trató con cirugía por vía laparoscópica.

\section{CASO CLÍNICO}

Paciente de 36 años de edad, sin antecedentes personales de interés salvo dos cesáreas, que fue remitida a nuestro servicio por dolor en fosa renal derecha.

En la radiografía simple de abdomen se evidencia una litiasis renal derecha y en la urografía intravenosa una acodadura del uréter derecho en su tercio superior sin otros hallazgos significativos. Asintomática, la paciente fue dada de alta. A los dos meses, nuevo ingreso por la misma clínica y nueva batería de pruebas: en la radiografía simple de abdomen se comprueba la existencia de una litiasis a nivel de uréter distal derecho y pequeñas calcificaciones en riñón derecho; la ecografía abdominal confirma lo hallado en la radiografía y además descarta la existencia de uropatía obstructiva. En todo momento las pruebas de laboratorio se encuentran dentro de la normalidad.

Se trata la litiasis ureteral mediante ureteroscopia y lasertricia persistiendo la clínica a pesar de la eficacia del tratamiento lo que motiva nuevo ingreso unos meses más tarde. Se realiza UIV donde se observa una ectasia ureteropielocalicial derecha hasta cresta iliaca (Fig. 1). En la TC los riñones son normales pero el uréter derecho está discretamente dilatado hasta el tercio distal donde se identifica un quiste ovárico de $1,5 \mathrm{~cm}$, que pudiera estar comprimiendo parcialmente el tercio distal del uréter (vista por el servicio de ginecología no precisa tratamiento alguno, solamente revisiones periódicas).

En la pielografía ascendente que se realiza se ve la ectasia ureteropielocalicial sin observar claramente una estenosis ureteral; se coloca un catéter doble $\mathrm{J}$ que es retirado a las 8 semanas sin apreciar la paciente mejoría clínica. Se decide hacer una resonancia magnética nuclear (RMN) que nos pueda aportar algún dato nuevo que justifique los sintomas; dicha RMN nos informa de una ectasia ureteral derecha por un posible síndrome de la vena ovárica (Figs. 2 y 3).

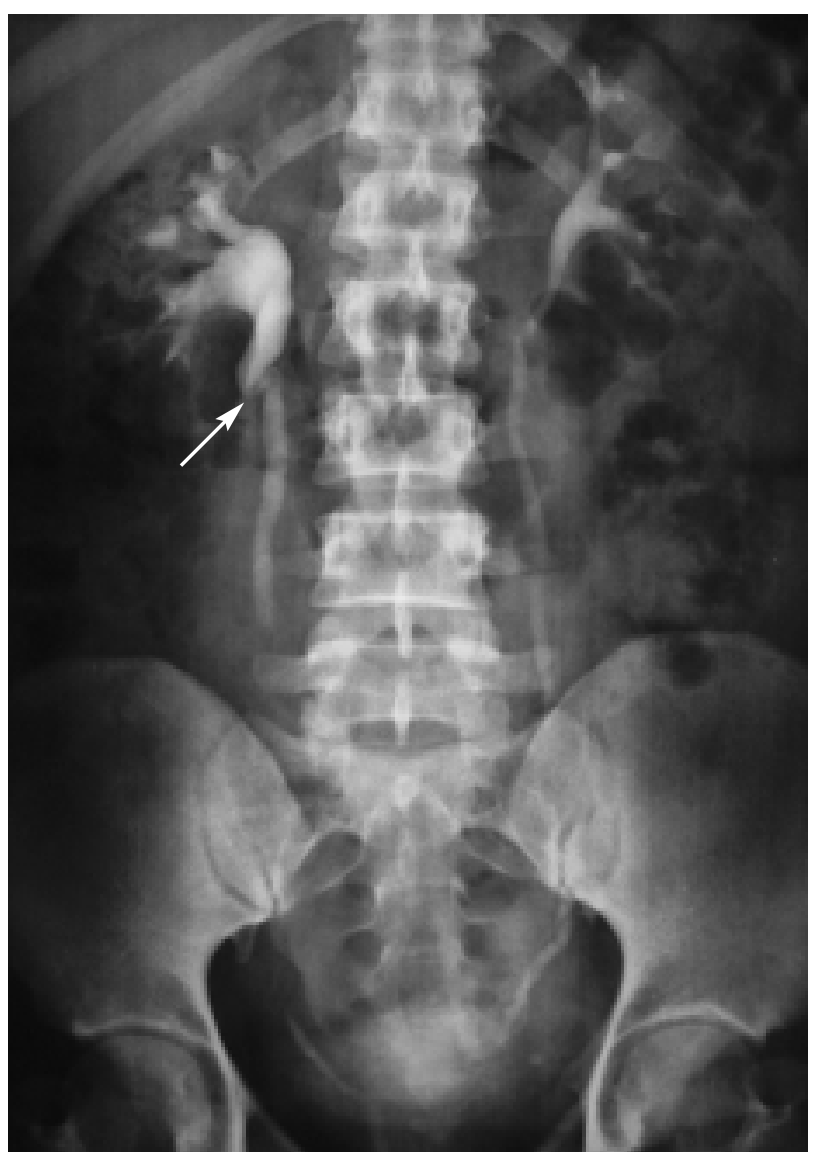

FIGURA 1. UIV: ectasia uretero- pielo-calicial derecha hasta cresta iliaca. Bucle ureteral proximal ( $)$.

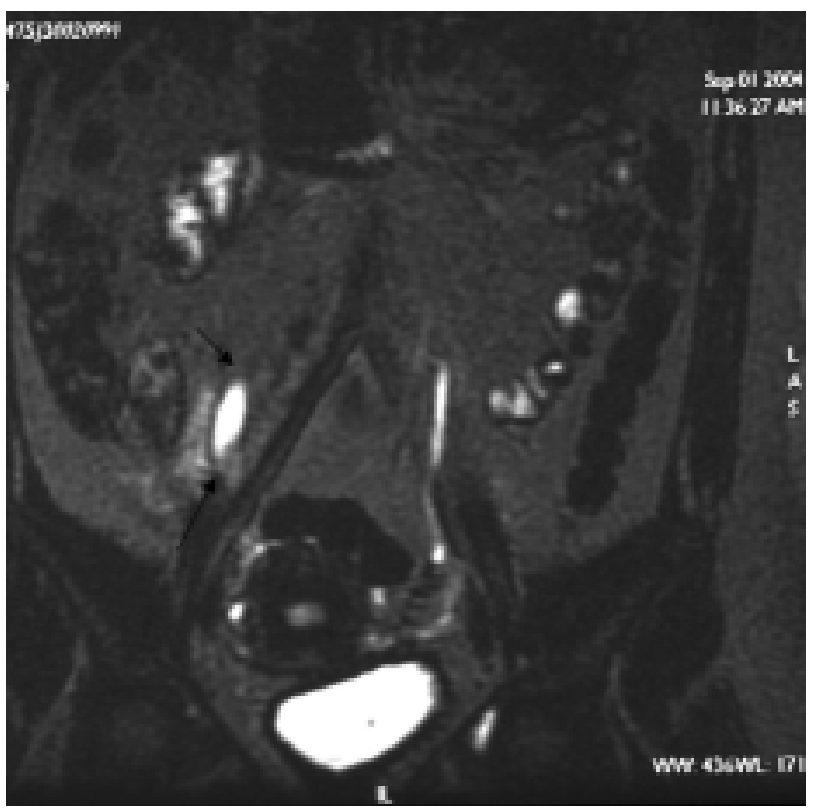

FIGURA 2. RMN: uréter derecho dilatado en su porción iliaca con una estenosis posterior que corresponde al cruce de la vena ovárica. 


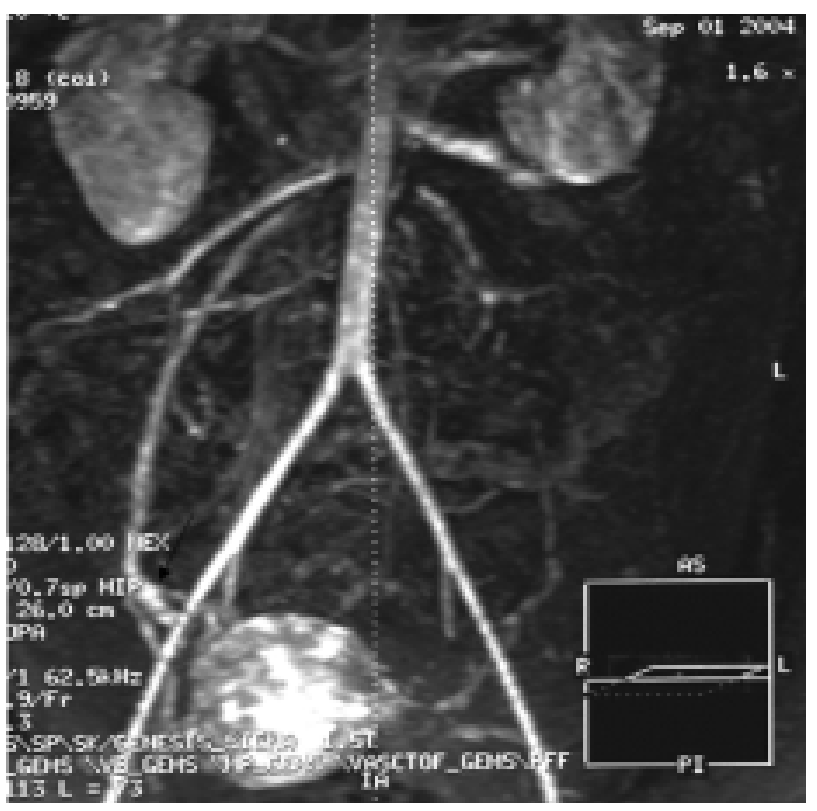

FIGURA 3. RMN: Vena gonadal que se bifurca a nivel de arteria iliaca donde realiza efecto de pinza sobre el uréter derecho provocando la obstrucción.

Se programa para intervención quirúrgica: ligadura de vena ovárica más ureterolisis derecha por vía laparoscópica transabdominal. La paciente es colocada en ligero decúbito lateral izquierdo. Insuflación abdominal con $\mathrm{CO}_{2}$ a través de una aguja de Veress y colocación de cuatro trócares, 2 de $5 \mathrm{~mm}$-(uno subesternal y otro en la zona lumbar entre la cresta iliaca y la $12^{\text {a }}$ costilla)- y 2 de $10 \mathrm{~mm}$-(uno en el ombligo, donde se coloca la óptica, y el otro suprapúbico)-. Tras la incisión de la línea de Toldt y decolación se procede a la identificación del uréter lumbar y seguimiento del mismo hasta observar su paso entre la arteria iliaca y la vena ovárica (Fig. 4), ésta última cruza por encima del uréter y se bifurca en 2 ramas que actúan a modo de pinza comprimiendo el uréter (Fig. 5). Se libera la vena ovárica y posteriormente se procede a la ligadura y sección de la misma (Fig. 6). Para finalizar, colocamos un drenaje tipo Redon sin aspiración a través del trócar lateral, se confirma la hemostasia, se evacúa el $\mathrm{CO}_{2}$ y se suturan los puertos de entrada. El postoperatorio cursa sin incidencias y la paciente es dada de alta al segundo día de la intervención.

En revisiones posteriores, a los 3 y 6 meses después de la cirugía, se encuentra totalmente asintomática y en la UIV de control ha desaparecido la ectasia y acodadura del uréter derecho.

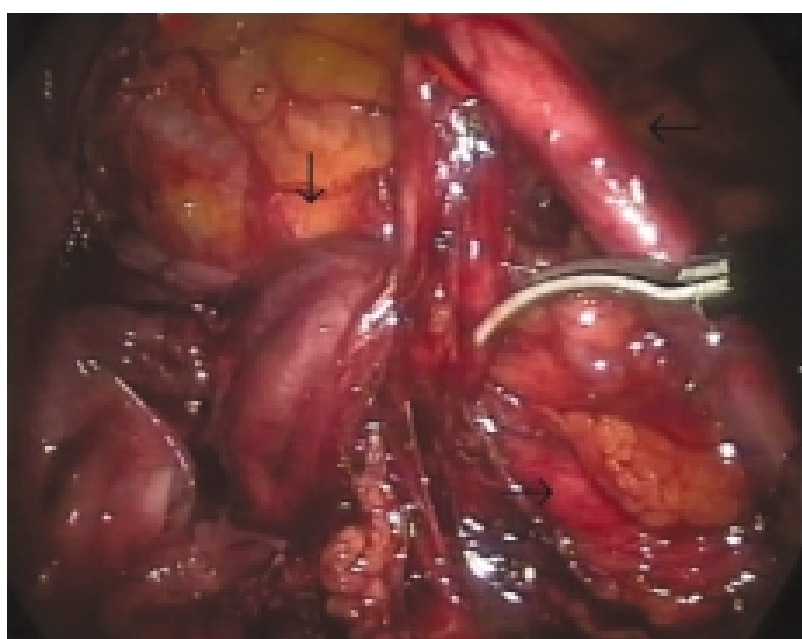

FIGURA 4. Se observa el uréter $(\leftrightarrow)$ pasando entre la arte ria iliaca $(\rightarrow$ y la vena ovárica $(\downarrow)$.

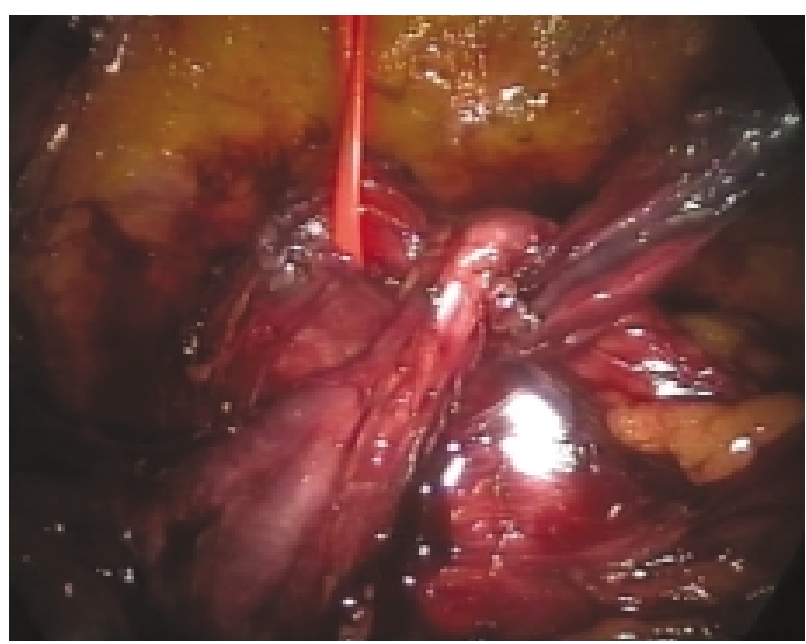

FIGURA 5. Vena ovárica cruzando por encima del uréter liberado.

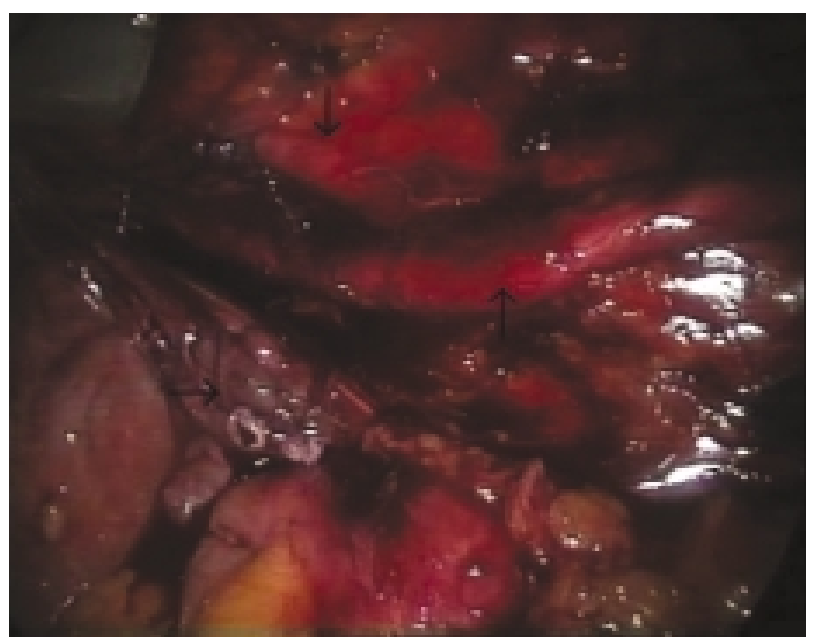

FIGURA 6. Ureterolisis finalizada. Se puede observar el uréter ( $($ ), la arteria iliaca $(\downarrow)$ y la vena ovárica ligada y seccionada $(\rightarrow)$. 


\section{DISCUSIÓN}

La obstrucción ureteral secundaria a su relación con la vena ovárica ha sido discutida por muchos autores desde la descripción inicial de Clark $^{3,4}$.

Dure-Smith ${ }^{4}$ publica en 1979 una revisión en la que concluye que no ha sido demostrada que la simple dilatación de la vena ovárica produzca obstrucción ureteral y que la dilatación existente en el síndrome de la vena ovárica es idéntica a la existente en todos los embarazos.

Durante el embarazo se produce un aumento del diámetro del uréter que llega a ser máximo en la semana 22 y que desaparece a las tres semanas del parto. Se ha demostrado que dicha dilatación no es debida a la presión ejercida por el útero y sí, a influencias hormonales. Asímismo existe un aumento del flujo en la vena ovárica junto con un aumento del diámetro de la misma por lo que no aumenta la presión. La involución de la vena es lenta y a veces no es completa ${ }^{5}$.

En su etiopatogenia se han involucrado numerosas causas:

a) Aumento de presión por una vena ovárica dilatada ${ }^{1,2,}$.

b) Existencia de una vena ovárica aberrante ${ }^{3}$.

c) Existencia de una vaina fibrosa ${ }^{4}$.

d) Tromboflebitis de la vena ovárica ${ }^{6,7}$.

e) Tumores que invaden la cava ${ }^{2,8}$.

El síndrome de la vena ovárica es más frecuente en el lado derecho; clínicamente se manifiesta como un cuadro agudo en embarazadas que consiste en una pielonefritis aguda o en una crisis renoureteral, o como un cuadro crónico en no embarazadas que se manifiesta como infección urinaria de repetición o como un dolor sordo y continuo en fosa renal, que frecuentemente se exacerba entre la ovulación y la menstruación ${ }^{3}$.

El diagnóstico de esta entidad es difícil debido a que no solemos relacionar la coincidencia en el tiempo del cuadro clínico de cólico nefrítico con el periodo premenstrual y menstrual. Generalmente, el diagnóstico, suele realizarse después de valorar la ecografía y urografía que demostrarían una dilatación ureteropiélica junto con signos de estenosis o "stop" a nivel ureteral en el lugar afectado por su relación con la vena ovárica. El nivel de afectación suele ser el uréter lumbar (L3-L4) o bien a nivel del anillo pélvico. La ureteropielografía retrógrada sólo se debería utilizar cuando la urografía intravenosa no demostrara el uréter dis$\operatorname{tal}^{9,10}$. En la actualidad la flebografía no suele emplearse de forma habitual ${ }^{10}$. En la década de los 80-90 este procedimiento se utilizaba con más frecuencia ya que en muchos casos trataban el síndrome de la vena ovárica con embolización percutánea para lo cual requerían de este procedimiento diagnóstico ${ }^{5}$.

En el caso presentado, la RMN nos reveló el diagnóstico observando cómo la vena ovárica, a nivel lumbar bajo, presenta una bifurcación que engloba al uréter ocasionando dilatación pieloureteral y signos de estenosis a dicho nivel.

El diagnóstico diferencial debe realizarse con otros procesos extrínsecos que puedan producir obstrucción ureteral ${ }^{11}$. La prueba diagnóstica más aconsejable sería una TAC. Cuando la clínica de dolor renal se altera con los cambios de posición se deberían considerar dos patologías: la ptosis renal (si el dolor aparece o se incrementa en la bipedestación) y el sindrome de la vena ovárica (si el dolor aparece con el decúbito) ${ }^{12}$.

Con respecto al tratamiento, dependiendo si la mujer está embarazada o no el planteamiento es distinto.

El tratamiento clásico ha sido quirúrgico mediante ureterolisis ${ }^{3}$. Dentro de la opción quirúrgica se puede abordar por vía abierta o mediante laparoscopia.

En los últimos años se han publicado varios artículos que comparan los resultados entre la cirugía abierta y la cirugía laparoscópica en el tratamiento del síndrome de la vena ovárica, concluyendo la menor morbilidad, menor estancia media hospitalaria, menor convalecencia del abordaje laparoscópico respecto al abierto ${ }^{13,14}$.

Como resultado del tratamiento quirúrgico (tanto abierto como por vía laparoscópica) mejora o desaparece la clínica de los pacientes, permaneciendo una imagen radiológica residual de dilatación del sistema excretor. No obstante, en algunos casos, existe regresión morfofuncional radiológica completa ${ }^{15}$.

En conclusión, toda paciente en edad fértil, con clínica de tipo cólico nefrítico, sobre todo del lado derecho, en la que se han descartado las causas obstructivas habituales, y que la clínica coincide en el tiempo con la menstruación 
o los días previos a ésta, se debería hacer diagnóstico diferencial con este cuadro clínico poco habitual de Síndrome de la Vena Ovárica. Confirmado dicho cuadro, el tratamiento más indicado en la actualidad sería la cirugía laparoscópica.

\section{REFERENCIAS}

1. Roberts JA, Dykhuizen RF. The ovarian vein syndrome. Surg Gynecol Obstet 1970;130:443-452.

2. Melnick RG and Bramwit DM. Bilateral ovarian vein syndrome. Am J Roentgenol Radium Ther Nucl Med 1971;113: 509-512.

3. Dure-Smith P. Ovarian syndrome: is it a myth?. Urology 1979;13:355-364.

4. Clark JC. The right ovarian vein syndrome. Emmett J.J. ed Clinical urography: An Atlas end Texbook of Roentgenologic Diagnosis. $2^{\mathrm{a}}$ ed. W.B. Saunders Company. Philadelphia 1964;1227-1236.

5. Moyano Calvo JL, Teba del Pino $\mathrm{F}$, Arellano Gañán R, Romero Tejada JC, Albacete Almodóvar P, Ruiz Zarate C, et al. Síndrome de la vena ovárica: tratamiento por embolización percutánea y revisión del mismo. Arch Esp Urol 1993; 46:802-806.

6. Coolsaet BL. Ureteric pathology in relation to right and left gonadal veins. Urology 1978;12:40-49.

7. Hubmer G. The ovarian vein syndrome. Eur Urol 1978;4 263-268.
8. Ashleigh RJ, Sambrook P. Case report: unilateral hidronephrosis following obstruction of the inferior vena cava by tumour thrombus. Clin Radiol 1991;44:130-131.

9. Monsalve Rodríguez M, Gómez Cisneros S y García Alonso J. Sindrome de la vena ovárica. Actas Urol Esp 1984;8:79-84.

10. Sanz Jaka JP, Mendivil Dacal J, Estébanez Zarranz J, Aldabe Villanueva J, Arocena Lanz F. Síndrome de la vena ovárica. Arch Esp Urol 1988;41:827-830.

11. Koch MO, Coussens D and Burnett L. The ovarian remnant syndrome and ureteral obstruction: medical management. J Urol 1994;152:158-160.

12. Marcovich R and Stuart Wolf Jr. Laparoscopy for the treatment of positional renal pain. Urology 1998;52:38-43.

13. Elashry OM, Nakada SY, Wolf JS Jr, Figenshau RS, Mc Dougall EM, Clayman RV. Ureterolysis for extrinsic ureteral obstruction: a comparision of laparoscopic and open surgical techniques. J Urol 1996;156:1403-1411.

14. Gettman MT, Yair Loton MD, Cadeddu J. Laparoscopic treatment of ovarian vein syndrome. JSLS 2003;7:257263.

15. Ferrero Doria R, Guzman Valls P, López Alba J, Tomas Ros M, Rico Galiano JL, Fontana Campiano LO. Síndrome de la vena ovárica. Actas Urol Esp 1996;20(4):380-383.

Dra. N. del Valle González.

E-mail: ndelvalle@telefonica.net

(Trabajo recibido el 4 de mayo 2005) 